\title{
Contributions to the development of search and rescue mobile robots
}

\author{
Marton Gyarmati ${ }^{1}$, Mihai Olimpiu Tătar ${ }^{1, *}$, and Francisc Kadar $^{1}$ \\ ${ }^{1}$ Department of Mechatronics and Machine Dynamics, Technical University of Cluj-Napoca, Cluj- \\ Napoca, Romania
}

\begin{abstract}
In this paper the authors present contributions to the development of search and rescue mobile robots. The first part of the paper describes the characteristics of search and rescue field. In the second part the authors presented the development and construction of an experimental prototype focusing on the locomotion systems for the search and rescue field and the results of the physical experiments done and the design and development of a proposed search and rescue mobile robot based on the lessons learned from the experiments. The third part contains the operation and control of the robot. The fourth section presents the simulation of the hybrid locomotion system of the proposed search and rescue mobile robot. The last part of the paper contains the development directions and conclusions.
\end{abstract}

\section{Introduction}

Search and rescue robots had a great development lately, especially after the appearance of the various catastrophes that humanity has faced. Among the most publicized catastrophes are those like the one from the WTC towers from USA or the hurricanes that devastated entire continents, but this type of robots are also used in catastrophes or local accidents that do not always reach the international press.

This type of robots provides more safety to the rescuer teams which arrive on the dangerous area because they have the possibility to operate fully autonomous or remote controlled. Consequently, small mobile robots are needed, which can move on rugged and unstructured terrain, and also on various obstacles of different forms [1].

Even if the general motivation for the urban search and rescue robotics field (U.S.A.R.) is to save lives and to protect the saviours, the main motivation for the models and characteristics of these robots depends very much on the possible tasks which have to be performed by them.

Some examples of the tasks that are encountered in this field are the following:

- searching is an activity that is carried out inside buildings, under rubble or rugged terrain in order to detect survivors or dangers that rescuers may encounter during rescue operations.

\footnotetext{
*Corresponding author: olimpiu.tatar@mdm.utcluj.ro
} 
- recognition and mapping provide to the rescuers an overview of the situation and generates a reference for the affected environment. The goal is to quickly cover an area of interest in the shortest possible time.

- inspections of structures are of two types. The interior inspections aim to determine the composition of the material debris to prevent the risk of a complete collapse of the affected buildings, while the external inspections aim to determine the degree of safety regarding the access of rescuers in these areas.

- medical evaluation allows verbal interactions between doctors and victims in order to provide first aid. It is also possible to transport medicines and liquids with the help of narrow tubes within four to ten hours, during which time the process of evacuation of victims can be organized [2].

- rescue is the term which represent the activities done after an incident that directly affects the survival of people. This includes localisation, check of health condition and removing of the survivors.

- rescue is also used as search and rescue term, which represents the activities and the teams which operates in this field, while rescue is mostly used to record a bigger disaster activity used to help the victims and teams.

The incidents can happen locally or can be a real disaster, which requires the involvement of special teams. The operations done during a disaster are split into 4 big phases: preparation, prevention, rescue and recovery. Preparation and prevention are the activities done before the incident, and the rescue and the recovery activities are done after the incident [3].

\section{Design and development of a search and rescue mobile robot}

The development of an optimal mobility system for robots provides a different solution to the problems that currently exist in this field. Using the desired system, the robot should be able to overcome various obstacles in emergency situations [4].

In order to make a good choice regarding the type of locomotion system used for the proposed search and rescue mobile robot, it is necessary to take into account robots' size, the environment and the type of the tasks performed [5].

First of all, a comparative study was made based on the specialized literature [6-10], to choose the locomotion system considered optimal for the proposed robot. A 3D CAD model was done, after which a prototype was executed. Following the physical tests, certain improvement needs were observed and these were taken into account when the new 3D model was designed for the proposed search and rescue mobile robot.

\subsection{The first constructive variant}

The first constructive variant proposed by the authors is a mobile robot with 4 TriStar locomotion units, of which 2 active and 2 passive units. The robot also contains proximity sensors, smoke sensor, LED bulbs, Arduino control board and battery. The size of the robot is $400 \mathrm{~mm} \times 872 \mathrm{~mm} \times 620 \mathrm{~mm}$ (Height $\times$ Length $\times$ Width) and can be used for rough terrain or buildings affected by disasters with the great advantage of overcoming various obstacles and stairs of certain sizes. It is known from the specialized literature about the advantages of using robots with locomotion systems with tracks for the rugged areas, but also the hybrid system proposed can successfully overcome the obstacles found in these environments [6]. We only called it a mobile robot because we focused more on the locomotion system that can be used successfully in the required domain.

The active TriStar unit consists of 3 legs positioned at 120 degrees, with 3 gears and a classic wheel at the end of the legs, and the passive TriStar unit is similar, the difference being that the 
passive wheel does not have a gear system and is not powered by a motor [11]. The steering is performed by differentially controlling the 2 front locomotion units (Fig. 1).

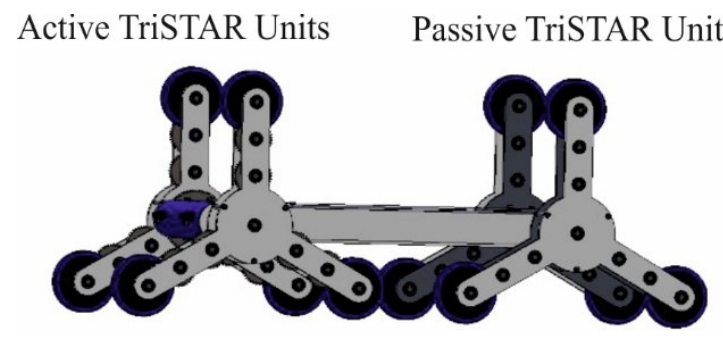

a)

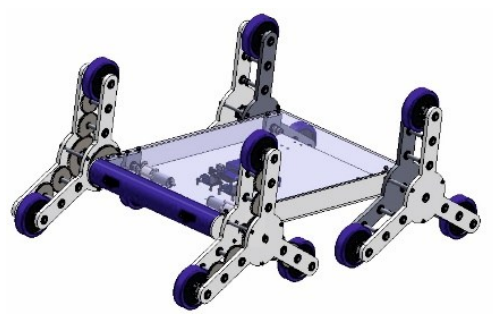

b)

Fig. 1. The first constructive variant $-3 \mathrm{D}$ model.

In order to be able to test the proposed locomotion units, the prototype shown in Fig. 2 was built. It uses for actuation two DC motors with reducer [12].

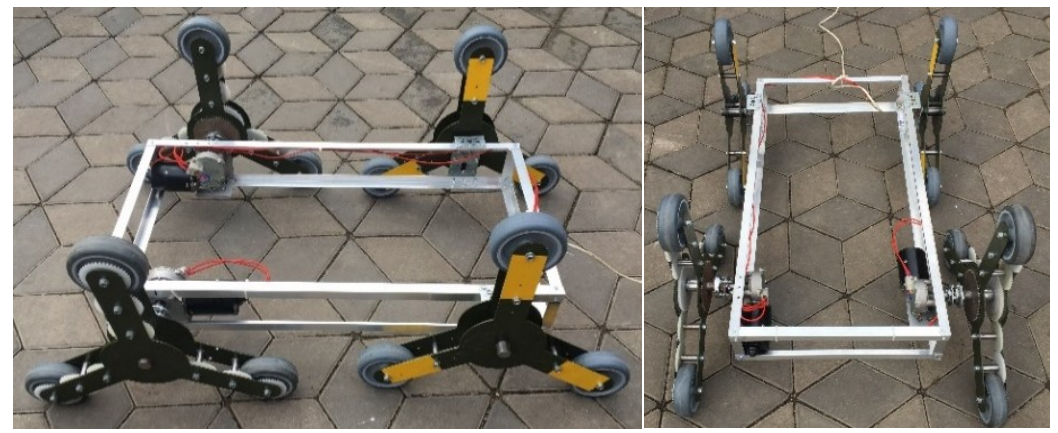

Fig. 2. Experimental prototype.

\subsubsection{Testing of the experimental prototype}

To test the locomotion process of the experimental prototype, a working area was used in which we found surfaces similar to those from the field of search and rescue.

Fig. 3 shows frames from one of the videos taken while the robot overcomes an obstacle and continues to move on the country road. Below are some images from the test and some conclusions that resulted from the experimental tests.

In the pictures from Fig. 4, different locomotion surfaces can be identified, such as: flat paved surface, gravel road, grassy terrain, muddy road, countryside road covered with different types of gravel similar to the rubble. The locomotion process of the experimental prototype was tested on the enumerated locomotion surfaces.

The conclusions gained from the experimental tests are the following:

- the search and rescue robots operate in difficult and harsh environments, involving the need for a versatile locomotion system that can adapt to different types of terrain;

-to avoid the wheel lock due to rubble or mud that could enter between the axles or the gear transmission system, it is recommended to seal the transmission system of the locomotion unit used;

-the robot's size may cause difficulties in its transportation process. It is recommended to reduce the size and weight of the robot in order to facilitate an easy transportation method, for example, inside a backpack;

-if the robot is expected to exceed obstacles such as stairs, the diameter of the wheels should be calculated in accordance with the dimensions of the stairs, otherwise the robot 
will not be able to overpass the stairs and remain blocked without the possibility of going further;

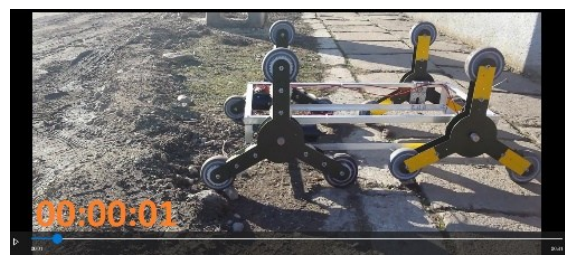

a)

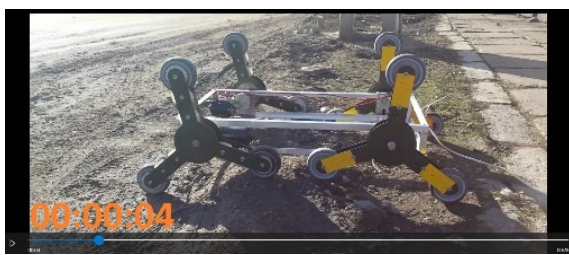

c)

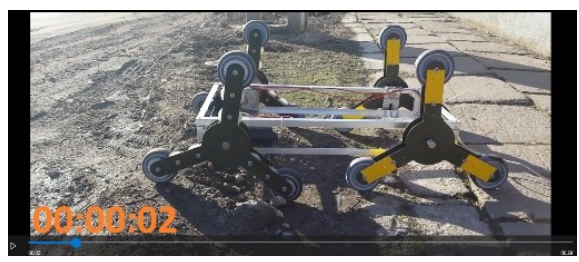

b)

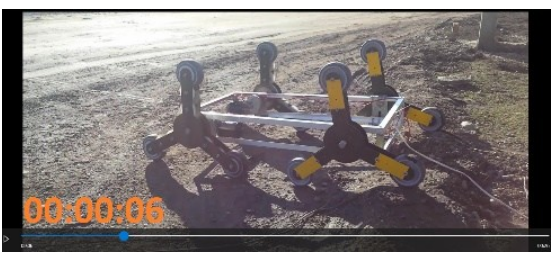

d)

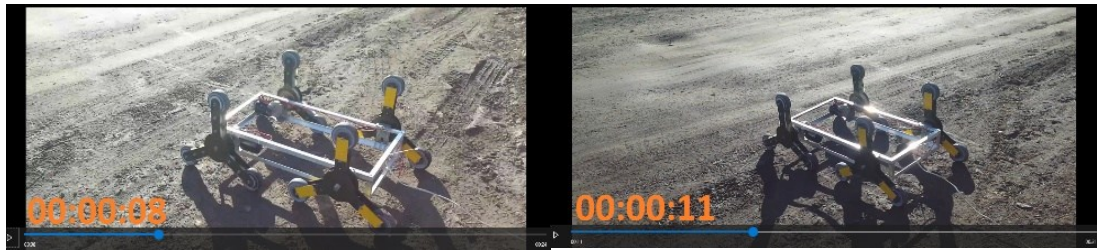

e)

f)

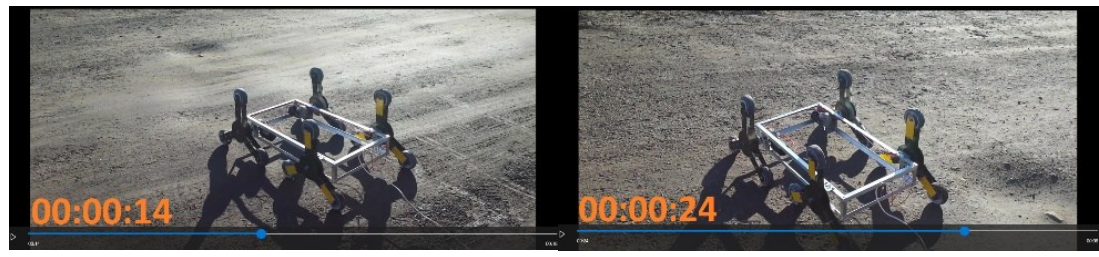

g)

h)

Fig. 3. Experimental prototype overcoming an obstacle.

- to avoid the possible technical problems during the search and rescue operations, such as short circuits, mechanical or water damage of the electronic components, it is recommended to hermetically seal the structure of the robot;

- increasing the wheel size of the TriStar units and modifying the robot platform to obtain a structure with a higher degree of adaptability to the specific characteristics of the locomotion surfaces;

- one possible improvement idea would be the energy supply with batteries, but the disadvantages would be the increase of weight of the robot's structure and the limited operational range, while the wire-powered version has limited mobility due to the power cord, but it offers unlimited power and operational range;

- to avoid the need for a second motor in order to obtain a proper functionality of the TriStar unit and the use of the possible locomotion methods of the robot: driving through the attached wheels, as well as through the TriStar system, it is proposed to use an odd number of gears in the transmission systems. 


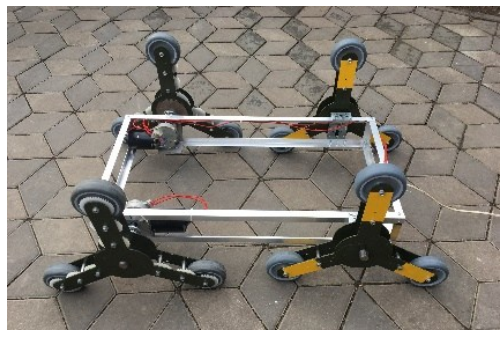

a)

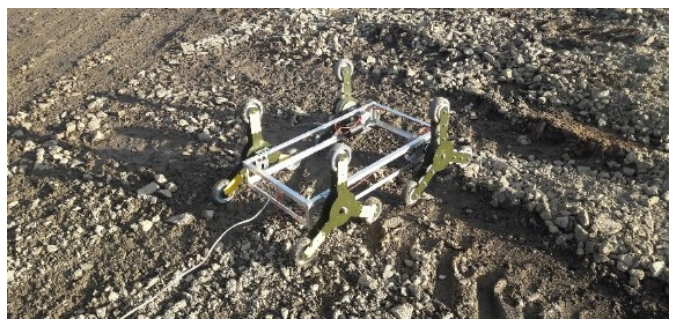

c)

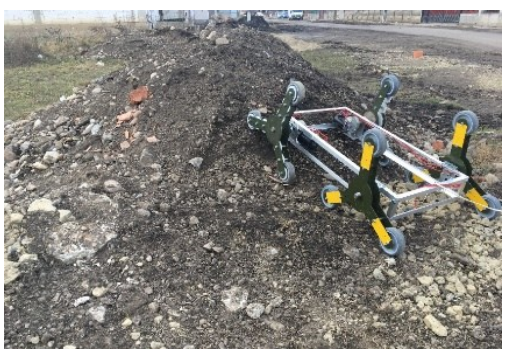

e)

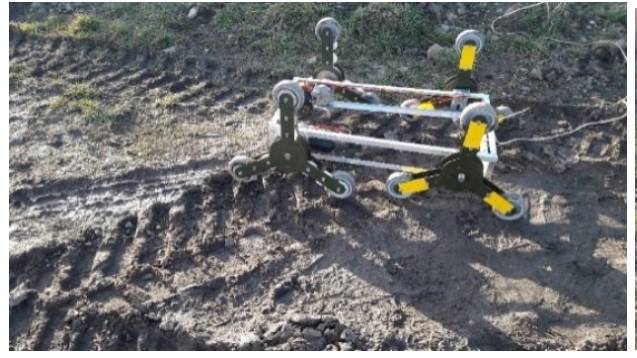

g)

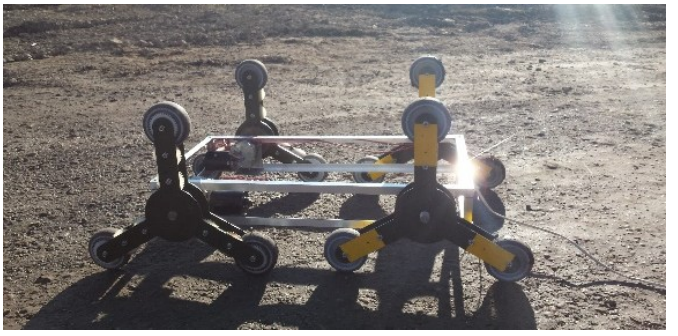

b)

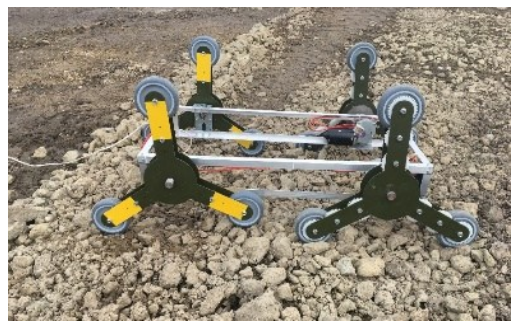

d)

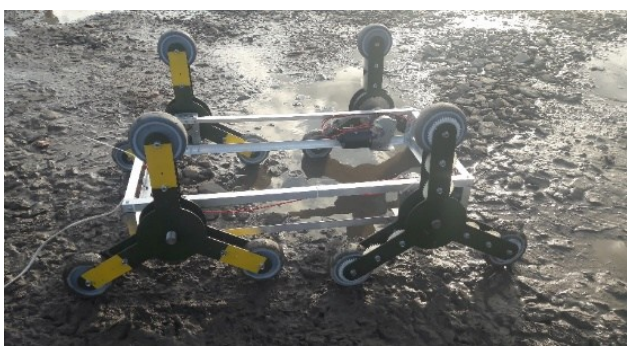

f)

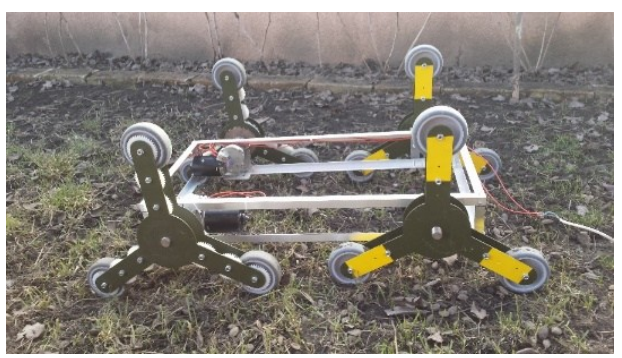

h)

Fig. 4. Images from outdoor testing the experimental prototype on different locomotion surfaces.

\subsection{Second constructive solution}

Based on the experiences with the prototype obtained using the first constructive solution, a mobile robot was developed for the search and rescue consisting of a hybrid locomotion system, containing 2 TriStar locomotion units and 2 classic wheels is proposed. This platform may also be used if is required with 4 conventional wheels or 4 TriStar locomotion units [13-16] (Fig. 5). 


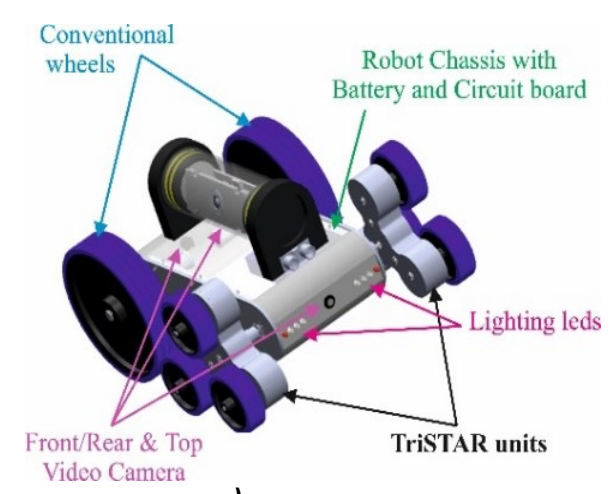

a)

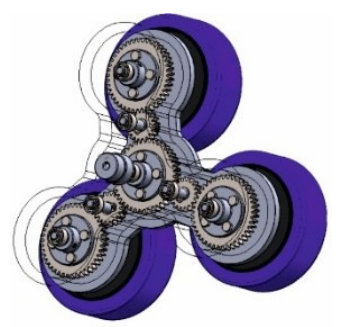

b)

Fig. 5. The 3D model for the second constructive solution and the TriStar locomotion unit's structure.

The proposed robot's dimensions are $400 \mathrm{~mm} \times 380 \mathrm{~mm} \times 180 \mathrm{~mm}$, so it's easy to transport. In order to fit into the required field, and also to be considered an autonomous mobile robot, this robot is equipped with 4 DC motors with integrated GHM-01 speed reducer, Arduino control board, multiple video cameras, LEDs, sensors and battery [14].

The locomotion systems have been improved by being hermetically sealed, the mud or rubble can no longer reach the gears and block the locomotion units.

Compared to the first built TriStar unit, the new unit shows improvements at the gear transmissions, its geometry allows us to change the locomotion modes: from advancing mode to automatic climbing mode $[11,15]$.

The new CAD model for the locomotion unit has been developed taking into account the problems encountered in the on-field experiments. The total height of the new locomotion unit is $200 \mathrm{~mm}$ and the possible height of the obstacles that can be overpassed is maximum $100 \mathrm{~mm}[13]$.

\section{Command and control of the proposed search and rescue robot}

The graphical user interface for the remote control of the robot and special modules used for performing search and rescue operations, was designed in the Matlab App Designer environment. The graphical user interface contains a main window with the purpose to control the robot's movement and real-time monitoring of the operating environment affected by natural disasters and accidents (Fig. 6). The main window allows access to secondary graphical user interfaces in order to use the functions of the special modules.

Since the robot was designed with modular system, to allow switching the front camera with a serial robotic arm, a secondary graphical user interface (Fig. 7) was created for controlling the functions of this special modules [13].

The camera module graphical user interface allows detailed real-time monitoring of the operating environment and the control of the light system. The light system and the video cameras can be switched on/off remotely to increase the robot's power autonomy and operational range [13].

The serial robotic arm module's graphical user interface allows the precise control of the angular position of the robot joints and gripper (Fig. 8). The gripper is equipped with an additional video camera and light source in order to allow precise and safe manipulation to remove obstacles and perform the visual inspection of the operating environment [13]. 


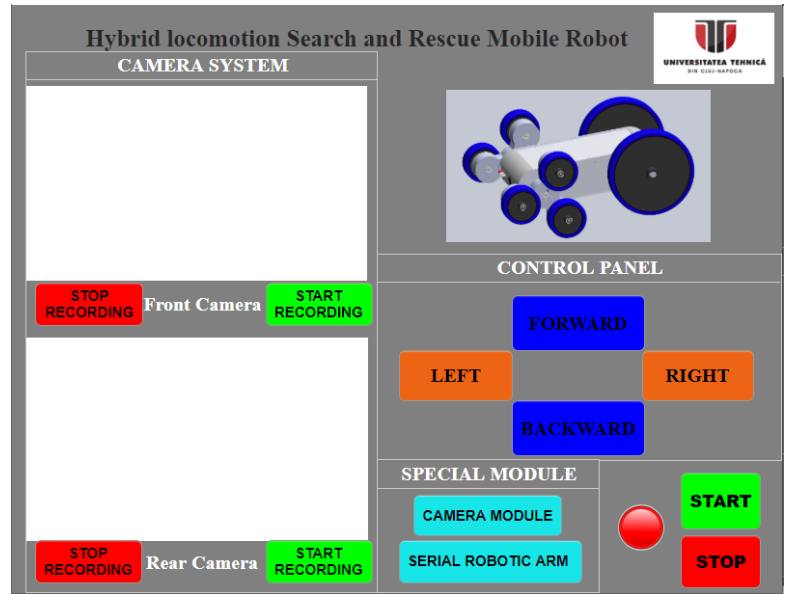

Fig. 6. The control interface of the mobile robot designed in Matlab.

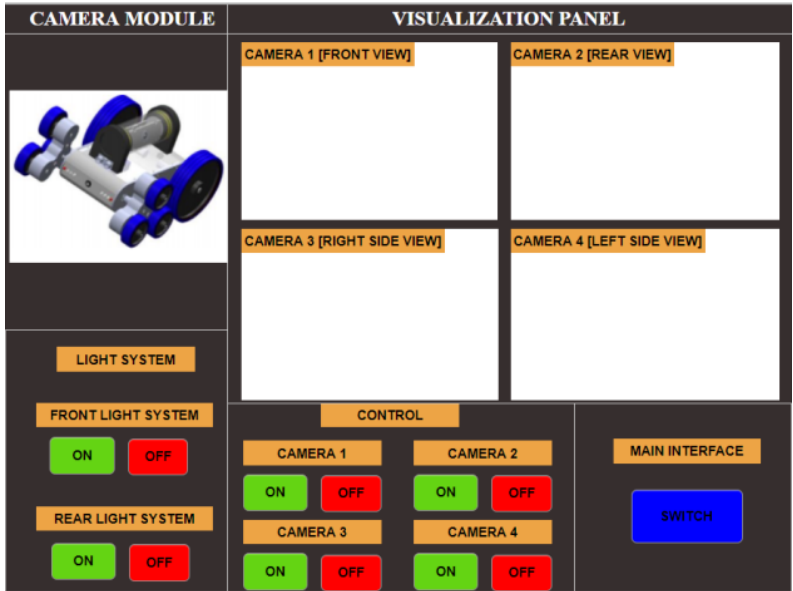

Fig. 7. Secondary graphical user interface for camera module control.

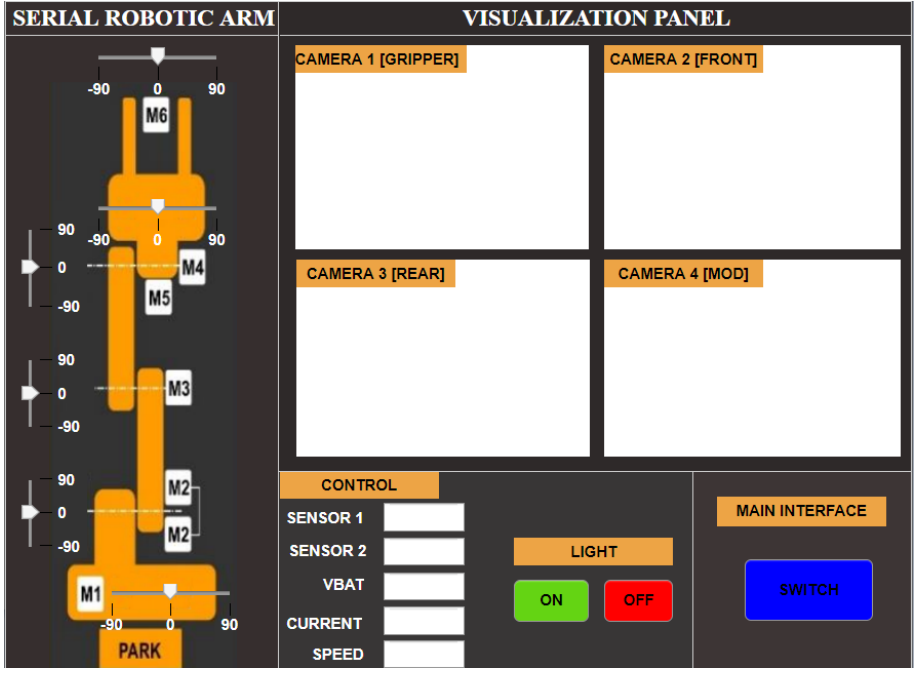

Fig. 8. Secondary graphical user interface for serial robotic arm control. 
The secondary graphical user interfaces designed with the purpose of controlling the functions of the special modules have implemented a button that allows switching to the primary control interface of the USAR robot.

\section{Simulation of the locomotion process for the proposed search and rescue mobile robot}

The simplified 3D model of the proposed search and rescue mobile robot was imported from the SolidWorks design environment into the Matlab programming environment using the Simscape Multibody Link plug-in. Matlab Simscape Multibody provides a 3D simulation environment of the locomotion process for the proposed robot design. The Hybrid locomotion Search and Rescue Mobile Robot subsystem contains the model parameters required for $3 \mathrm{D}$ visualization of the proposed robot locomotion process. In order to simulate the locomotion process, the moving elements of the model are controlled by the values provided to the actuators in Matlab Simulink (Fig. 9). The motion equation for this robot with hybrid system can be determined with Lagrange's equation [16].

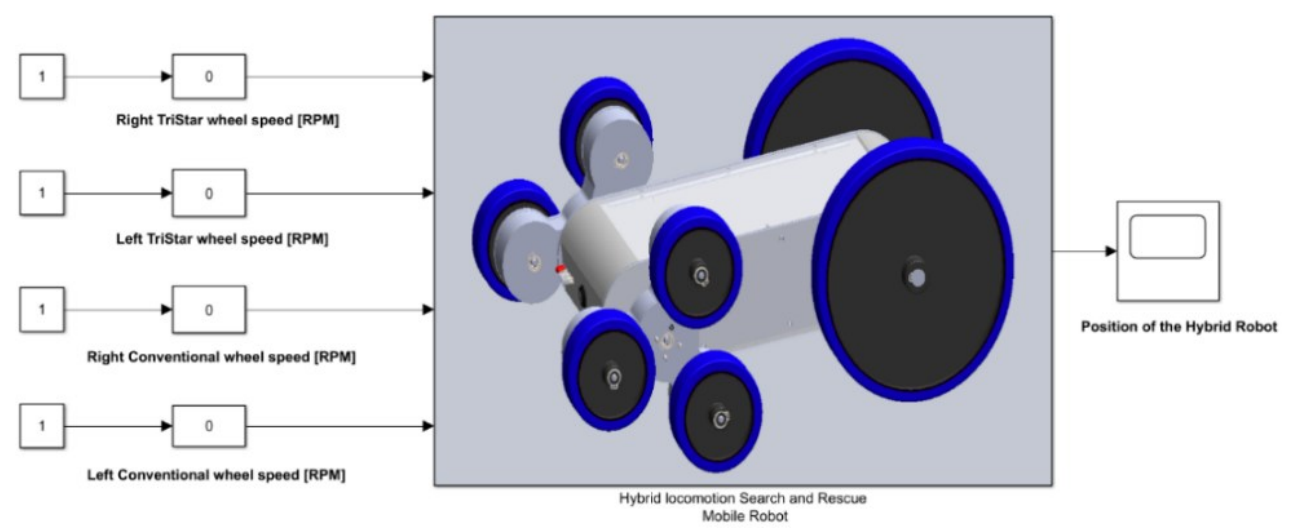

Fig. 9. The proposed USAR robot with hybrid locomotion in Matlab.

The 3D model of the search and rescue robot with a hybrid locomotion system imported into Matlab is simplified, contains only the moving elements and the structure of the robot (Fig. 10).

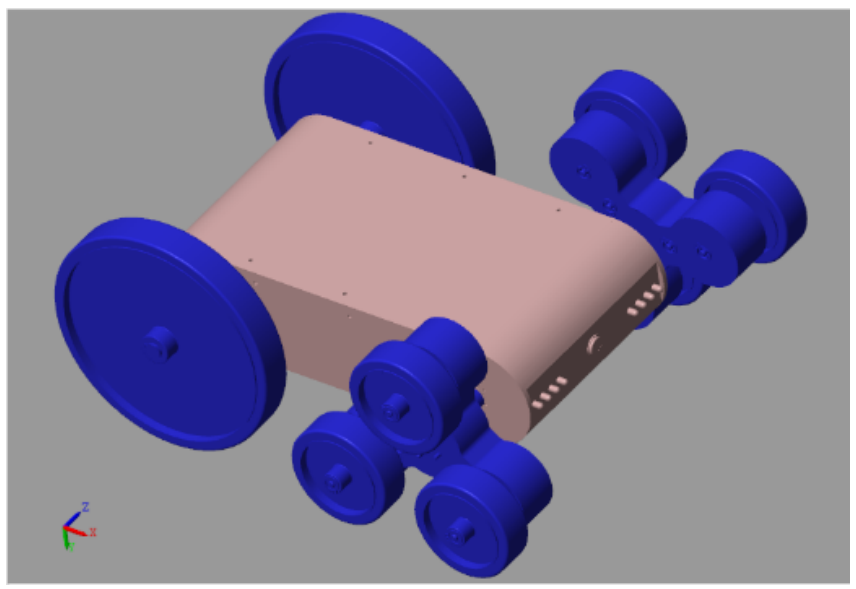

Fig. 10. The 3D model of the proposed USAR robot in Matlab. 
In Matlab was performed the simulation of the dynamic behaviour for the locomotion process of the proposed USAR mobile robot equipped with a hybrid system for locomotion. The proposed locomotion process is simulated for different locomotion surfaces found in the search and rescue operations.

The locomotion process and the behaviour of the proposed robot was simulated for the following types of terrain and locomotion surfaces:

- flat locomotion surface (Fig. 11. a);

- uneven terrain (Fig. 11. b);

- stairs climbing process (Fig. 11. c);

- process of descending the stairs (Fig. 11.d).

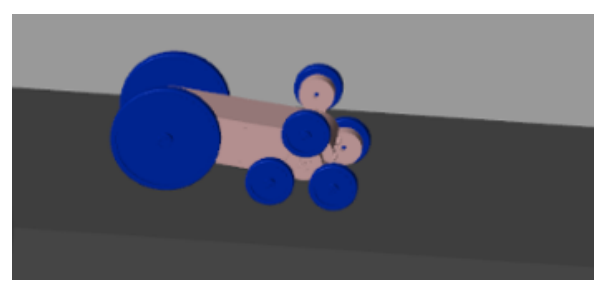

a)

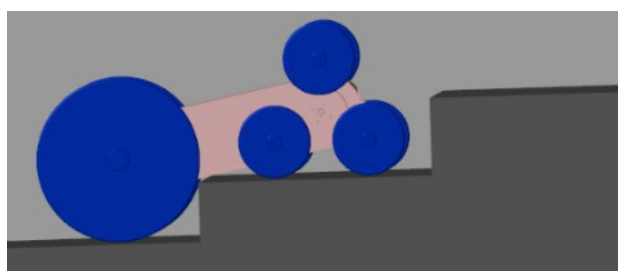

c)

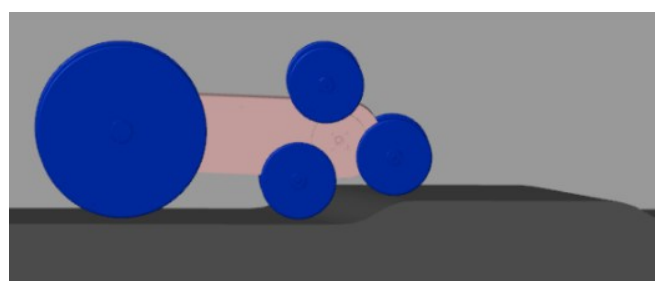

b)

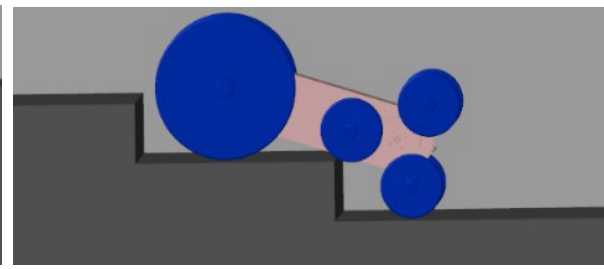

d)

Fig. 11. Simulation of the locomotion process for the proposed search and rescue mobile robot.

The simulation of the locomotion process performed in Matlab allows us to 3D visualize the behaviour of the proposed search and rescue robot on different locomotion surfaces, types of terrain and environmental conditions.

\section{Development directions}

Although the proposed mobile robot can be included in the search and rescue field, knowing that this robot is equipped with 4 locomotion units, in the future, it's proposed to implement a suspension system for the locomotion units in order to improve the adaptability to rough and uneven terrains and to avoid the chassis blocking on some obstacles. Additional improvement could be also to add an articulation on the middle of the structure to increase more the flexibility and adaptability of the robot.

A new experimental prototype will be developed, simulations and field tests will be performed. Based on the future results of the simulations additional improvements will be implemented if is necessary.

\section{Conclusions}

Search and rescue mobile robots provide greater safety for rescue teams arriving on the scene, so it is very important to take into account, when choosing the robot for a certain 
type of intervention the possible tasks to be performed, the characteristics of the environment and weather conditions.

The authors present a search and rescue mobile robot equipped with a hybrid locomotion system which contains 2 TriStar locomotion units and 2 classic wheels. The proposed model is an improved constructive solution of the prototype used to perform the experimental research on similar surfaces to those found in the field of search and rescue operations.

\section{References}

1. P. Labenda, T. Sadek, L. Heckes, 5th IFAC Sym. Mech. Syst., (2010)

2. J.A. Barbera, C. DeAtley, A.G. Macintyre, Fire Eng., 148, 88-92, (1995)

3. R. R. Murphy, S. Tadokoro, D. Nardi, A. Jacoff, Paolo Fiorini, H. Choset, A. M. Erkmen, Springer Handbook of Robotics, (Springer, Berlin, Heidelberg, 2008)

4. K. Hasnan, Q. Bakhsh, A. Ahmed, B. Das and S. Ayub, ICGCE (2015)

5. V. Zamanov, A. Dimitrov, RAM, (2011)

6. G. Quaglia, L. Bruzzone, G. Bozzini, R. Oderio, R. P. Razzoli, Industrial Robot: An International Journal, 38(3), 282 - 291, (2011)

7. R. Bogue, Industrial Robot: An International Journal 43(2), 138-143, (2016)

8. B. Choi, W. Lee, G. Park, Y. Lee, J. Min, S. Hong, J. Field Rob. 36(4), 656-676, (2019)

9. https://www.google.com/url?sa $=\mathrm{t} \& \mathrm{rct}=\mathrm{j} \& \mathrm{q}=\& \mathrm{esrc}=\mathrm{s} \&$ source $=$ web\& $\mathrm{cd}=\& \mathrm{ved}=2 \mathrm{ahUKEwjng}-$ XgkcrwAhWawQIHHfpgByUQFjAAegQIAxAD\&url=https\%3A\%2F\%2Fetd.ohiolink.edu\%2F apexprod\%2Frws_etd\%2Fsend_file\%2Fsend\%3Faccession\%3Dcase $1417782329 \% 26$ disposition \%3Dattachment\&usg=AOvVaw3H5OKEIPUFhJVeinIOFIr1, [Accessed 15.05.2021]

10. L. M. Smith, R. D. Quinn, K. A. Johnson, W. R. Tuck, IROS, (2015)

11. M.O. Tătar, M. Gyarmati, IOP Conf. Series - ACME (2018)

12. https://docs-emea.rs-online.com/webdocs/032c/0900766b8032c121.pdf, [Accessed 01.05.2021]

13. M. Gyarmati, M.O. Tătar, C. Rusu, New Advances in Mechanisms, Mechanical Transmissions and Robotics, MTM \& Robotics 2020 (Springer, Cham, 2021)

14. https://www.electrokit.com/uploads/productfile/41002/ghm01.pdf, [Accessed 13.03.2021]

15. M. Gyarmati, M.O. Tătar, Robotica Management 24(1), 8-1 (2019)

16. M. Gyarmati, M.O. Tătar, ACME (2020) 